\title{
Minimally invasive mitral valve surgery is associated with equivalent cost and shorter hospital stay when compared with traditional sternotomy
}

\author{
Pavan Atluri, MD, Robert L. Stetson, MHA, George Hung, BA, Ann C. Gaffey, MD, Wilson Y. Szeto, MD,
} Michael A. Acker, MD, and W. Clark Hargrove, MD

\begin{abstract}
Objective: Mitral valve surgery is increasingly performed through minimally invasive approaches. There are limited data regarding the cost of minimally invasive mitral valve surgery. Moreover, there are no data on the specific costs associated with mitral valve surgery. We undertook this study to compare the costs (total and subcomponent) of minimally invasive mitral valve surgery relative to traditional sternotomy.
\end{abstract}

Methods: All isolated mitral valve repairs performed in our health system from March 2012 through September 2013 were analyzed. To ensure like sets of patients, only those patients who underwent isolated mitral valve repairs with preoperative Society of Thoracic Surgeons scores of less than 4 were included in this study. A total of 159 patients were identified (sternotomy, 68; mini, 91). Total incurred direct cost was obtained from hospital financial records.

Results: Analysis demonstrated no difference in total cost (operative and postoperative) of mitral valve repair between mini and sternotomy $(\$ 25,515 \pm \$ 7598$ vs $\$ 26,049 \pm \$ 11,737 ; P=.74)$. Operative costs were higher for the mini cohort, whereas postoperative costs were significantly lower. Postoperative intensive care unit and total hospital stays were both significantly shorter for the mini cohort. There were no differences in postoperative complications or survival between groups.

Conclusions: Minimally invasive mitral valve surgery can be performed with overall equivalent cost and shorter hospital stay relative to traditional sternotomy. There is greater operative cost associated with minimally invasive mitral valve surgery that is offset by shorter intensive care unit and hospital stays. ( $\mathrm{J}$ Thorac Cardiovasc Surg 2016;151:385-8)

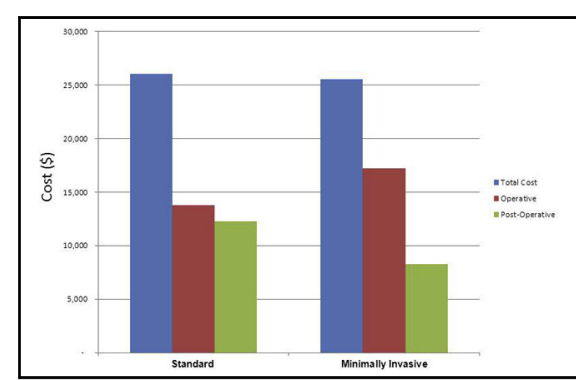

Cost associated with standard sternotomy and minimally invasive mitral valve repair.

\section{Central Message}

Total, operative, and postoperative costs were assessed for mitral valve repair performed by sternotomy and minimally invasive means.

Perspective

Economic concerns should not limit the use of minimally invasive mitral valve surgery. Minimally invasive mitral valve surgery can be performed with excellent outcomes in appropriately selected patients, which should also not limit application of this approach.

See Editorial Commentary page 389.
Minimally invasive mitral valve surgery has become increasingly prevalent. Several studies demonstrating equivalent operative results with excellent long-term

\footnotetext{
From the Division of Cardiovascular Surgery, Department of Surgery, University of Pennsylvania, Philadelphia, Pa.

Supported by the University of Pennsylvania, Department of Surgery.

Received for publication May 5, 2015; revisions received July 30, 2015; accepted for publication Aug 29, 2015; available ahead of print Sept 30, 2015.

Address for reprints: Pavan Atluri, MD, Director, Minimally Invasive and Robotic Cardiac Surgery, Director, Heart Transplantation and Mechanical Circulatory Support Program, Division of Cardiovascular Surgery, Department of Surgery, University of Pennsylvania, 6 Silverstein Pavilion, 3400 Spruce St, Philadelphia, PA 19104 (E-mail: pavan.atluri@uphs.upenn.edu).

0022-5223/\$36.00

Copyright $(2016$ by The American Association for Thoracic Surgery http://dx.doi.org/10.1016/j.jtcvs.2015.08.106
}

outcomes, namely freedom from recurrent mitral regurgitation, have provided support for this approach to the mitral valve. ${ }^{1}$ It has been demonstrated repeatedly that minimally invasive mitral valve repair is associated with longer operative and cardiopulmonary bypass times. $^{2}$ One would expect that with the increased need for reusable and disposable operative equipment (tissue retractors, cannulas, endoaortic balloons), there would

Scanning this $\mathrm{QR}$ code will take you to the article title page. 


\section{Abbreviations and Acronyms \\ $\mathrm{ICU}=$ intensive care unit \\ $\mathrm{IQR}=$ interquartile range}

be greater cost associated with this approach. On the other hand, numerous groups, including ours, have demonstrated shorter intensive care unit (ICU) and hospital stays associated with a minimally invasive technique. Unfortunately, there are minimal data in the literature regarding the economic considerations of minimally invasive mitral valve surgery. We undertook this study to evaluate the health care costs associated with minimally invasive mitral valve surgery relative to a traditional sternotomy approach. We hypothesized that minimally invasive mitral valve surgery could be performed with equivalent cost and outcomes to that performed through a sternotomy.

\section{METHODS \\ Study Design}

To assess the cost associated with either minimally invasive (right minithoracotomy) or sternotomy approaches to mitral valve repair, all patients who underwent isolated mitral valve repair at the University of Pennsylvania from March 2012 through September 2013 were evaluated. To ensure equivalent cost comparison, only isolated mitral valve repairs were taken into account. Patients who underwent concomitant procedures, such as ablation procedures for atrial fibrillation, tricuspid valve repair, and coronary artery bypass grafting, were excluded. Because of the cost and variability of expense associated with mitral valve prosthesis, mitral valve replacements were also excluded. In addition, high-risk patients (Society of Thoracic Surgeons risk score $>4$ ) were excluded from this analysis to have "like" subsets. Robotic repairs were also excluded. One hundred fifty-nine patients were identified who fit these criteria (sternotomy, $\mathrm{n}=68$; minimally invasive, $\mathrm{n}=91$ ).

Complete operative and postoperative costs associated with mitral valve repair were individually gathered from institutional billing records. Only direct hospital costs were used in this analysis; indirect costs and overhead were not incorporated. The cost components were grouped according to major subgroups for each patient and then further averaged according to the approach. Standard preoperative demographic and operative variables were compared between groups with our institutional Society of Thoracic Surgeons database. Postoperative outcomes and major complications (death, wound infection, stroke, reoperation for bleeding, atrial fibrillation, hospital and ICU stays, and survival) were also analyzed. The University of Pennsylvania Institutional Review Board approved these data for use in research, with a waiver of patient consent.

\section{Operative Approach}

All patients who underwent a minimally invasive mitral valve operation through a limited right thoracotomy with endoscopic assistance were incorporated into the minimally invasive cohort. Aortic occlusion was achieved with an endoaortic crossclamp (Edwards Lifesciences Corporation, Irvine, Calif) in most cases $(81 \%)$; in the remainder of the operations, a Chitwood clamp was used. We performed the minimally invasive operation with a limited right anterior thoracotomy and femoral cannulation, as previously described. ${ }^{3,4}$ For the sternotomy subgroup, the mitral valve was approached through a standard full sternotomy with standard central bicaval cannulation. Standard and similar operative repair techniques were used for both approaches.

In the setting of reoperative surgery in the minimal access cohort, the operation was performed through a similar right anterior thoracotomy. Once cardiopulmonary bypass was established through the femoral vessels, the lung was dissected free of the right lateral pericardium and the pericardium was opened. If necessary, intrapericardial adhesions were dissected free. The endoaortic balloon was used for aortic occlusion. The mitral valve was exposed through the interatrial groove, and the operation was conducted in standard fashion.

\section{Statistical Analysis}

Continuous variables are expressed as the median with interquartile range (IQR), and categorical variables are presented as proportions. Costs are expressed as mean \pm SD. Differences between groups were assessed with the Fisher exact test for categorical variables, the independent Student $t$ test for normally distributed continuous variables, and the Mann-Whitney $U$ test for nonparametrically distributed values. All tests were 2-tailed. The statistical analysis was performed with IBM SPSS Statistics (version 20.0; IBM Corporation, Armonk, NY).

\section{RESULTS \\ Preoperative Variables}

There was no difference in median preoperative Society of Thoracic Surgeons score between the minimally invasive and sternotomy cohorts (0.47; IQR, 0.36-0.95 vs 0.61 ; IQR, $0.34-1.35 ; P=.25$ ), demonstrating equivalent operative risks. Additionally, there was no difference in age (59 years; IQR, 52-66.5 years vs 57 years; IQR, 47-69 years; $P=.5)$, sex $(39.6 \%$ vs $38.2 \%$ female; $P>.999)$, or ethnicity $(87.9 \%$ vs $91.1 \%$ white; $P=.8)$ between minimally invasive and sternotomy cohorts, respectively. An equivalent percentage of patients in each group had a previous sternotomy $(8.6 \%$ vs $9.6 \%$; $P=.8)$.

\section{Operative, Postoperative, and Total Costs Associated With Mitral Valve Repair}

Analysis of total direct cost demonstrated equivalent costs between minimally invasive and sternotomy cohorts $(\$ 25,515 \pm \$ 7598$ vs $\$ 26,049 \pm \$ 11,737 ; P=.74)$. There was a higher operative cost associated with the minimally invasive cohort than with the sternotomy cohort $(\$ 17,246 \pm \$ 3823$ vs $\$ 13,786 \pm \$ 2594 ; P<.0000001)$. This was offset by significantly lower postoperative costs for the minimally invasive cohort $(\$ 8268 \pm \$ 6501$ vs $\$ 12,263 \pm \$ 10,836 ; P=.008)$.

Breakdown of the operative costs revealed the following major subcategories (minimally invasive vs sternotomy): operative supplies, $\$ 7539 \pm \$ 3577$ versus $\$ 5350 \pm \$ 1950$ $(P=.0000002)$; operative time, $\$ 3595 \pm \$ 698$ versus $\$ 3120 \pm \$ 650(P=.00002)$; perfusion, $\$ 3071 \pm \$ 619$ versus $\$ 2125 \pm \$ 619(P<.000001)$; nursing, $\$ 1685$ versus $\$ 1636 \pm \$ 287(P=.2)$; laboratory and blood bank, 
$\$ 716 \pm \$ 307$ versus $\$ 827 \pm \$ 598(P=.2)$; and pharmacy, $\$ 371 \pm \$ 195$ versus $\$ 447 \pm \$ 319(P=.09)$. Similarly, analysis of postoperative costs revealed the following major subcategories (minimally invasive vs sternotomy): intensive care nursing and board, $\$ 1593 \pm \$ 3854$ versus $\$ 2602 \pm \$ 4214(P=.1)$; step-down and floor nursing and board, $\$ 4305 \pm \$ 1636$ versus $\$ 4944 \pm \$ 2126(P=.04)$; pharmacy, $\$ 768 \pm \$ 1234$ versus $\$ 1428 \pm \$ 2165$ $(P=.03)$; cardiology (electrophysiology and echocardiography), $\$ 118 \pm \$ 758$ versus $\$ 1198 \pm \$ 4485(P=.05)$; ancillary (occupational, physical, and respiratory therapy), $\$ 535 \pm \$ 628$ versus $\$ 854 \pm \$ 894(P=.01)$; laboratory and blood bank, $\$ 511 \pm \$ 703$ versus $\$ 814 \pm \$ 854$ $(P=.02)$; and radiology, $\$ 361 \pm \$ 249$ vs $\$ 369 \pm \$ 291$ $(P=.9)$. There was a higher rate of pacemaker implantation in the sternotomy cohort $(\mathrm{n}=6)$ than in the minimally invasive group $(\mathrm{n}=1)$, which accounted for the significantly increased cardiology costs. Additional ancillary and laboratory costs resulted from the longer hospital stay with additional rehabilitation services and blood work.

\section{Outcomes Associated with Mitral Valve Repair Stratified by Approach}

As expected, cardiopulmonary bypass (118 minutes; IQR, 87-145 minutes vs 89 minutes; IQR, 72-120 minutes; $P=.0008$ ) and crossclamp (88 minutes; IQR, 56.5-109 minutes vs 66 minutes; IQR, 52-77 minutes; $P=.001)$ times were longer with the minimally invasive approach than with sternotomy. There were no differences in major complications, including stroke, atrial fibrillation, wound infection, renal failure, aortic dissection, and prolonged ventilation, between the groups. Notably, there were no cases of aortic dissection associated with femoral cannulation as used for the minimally invasive cohort. There were statistically significantly shorter median ICU stay ( 1 days; IQR, 1-2 vs 1.5 days; IQR, 1-3 days; $P=.05$ ) and hospital stay (6 days; IQR, 5-7 days vs 7 days; IQR, 6-9 days; $P=.005$ ) associated with the minimally invasive approach than with sternotomy. There were no deaths at 30 days in either cohort (Table 1).

\section{DISCUSSION}

As we have demonstrated in this study, minimally invasive mitral valve surgery can be performed with equivalent cost and outcomes as those seen in patients who underwent the same procedure through a sternotomy. As would be expected, in our experience we had shorter hospital and ICU stays, which equated to a significant total cost saving. This cost saving was offset by the higher operative costs that were noted with minimally invasive
TABLE 1. Postoperative outcomes after mitral valve repair, stratified by operative approach

\begin{tabular}{lccc}
\hline & $\begin{array}{c}\text { Minimally } \\
\text { invasive }\end{array}$ & Sternotomy & $\begin{array}{c}\boldsymbol{P} \\
\text { value }\end{array}$ \\
\hline Reexploration for bleeding (\%) & 0 & 0 & $>.999$ \\
Wound infection & 0 & $1(1.5 \%)$ & $>.999$ \\
Stroke & $1(1.1 \%)$ & $1(1.5 \%)$ & $>.999$ \\
Renal failure & 0 & 0 & $>.999$ \\
Aortic dissection & 0 & 0 & $>.999$ \\
Ventilatory support >24 h & $5(5.5 \%)$ & $6(8.2 \%)$ & .5 \\
Postoperative atrial fibrillation & $26(28.6 \%)$ & $29(42.6 \%)$ & .2 \\
ICU stay (d, mean and IQR) & $1(1-2)$ & $1.5(1-3)$ & .05 \\
Hospital stay (d, mean and IQR) & $6(5-7)$ & $7(6-9)$ & .005 \\
Readmission at 30 d & $3(3.2 \%)$ & $4(5.5 \%)$ & .3 \\
Mortality at 30 d & 0 & 0 & $>.999$ \\
\hline
\end{tabular}

Data represent number and percentage of patients unless otherwise specified. $I C U$, Intensive care unit; $I Q R$, interquartile range.

mitral valve repair. In our experience, there is a significantly higher cost associated with minimally invasive mitral valve surgery. This is largely related to the endoaortic balloon that is our aortic occlusion technique of choice. Additional operative cost is associated with longer operative times, with increased associated nursing, operating room, and perfusion costs. Moreover, the minimally invasive soft tissue retractors, cannulas, and ports, although rather inexpensive in comparison are additional contributors to cost. Overall, there is an equivalent cost between the approaches.

This study corroborates the findings of 2 previous reports that found cost savings associated with a minimally invasive approach. Iribarne and colleagues, ${ }^{5}$ investigating the Columbia University experience, noted statistically significant cost savings with minimally invasive mitral valve repair relative to sternotomy. Although the results of Iribarne and colleagues ${ }^{5}$ are similar to the data we have presented, there are significant differences that are notable. Namely, their cannulation technique was direct aortic cannulation with a Chitwood clamp. Although this is a very safe and acceptable approach, our preference is for femoral cannulation with an endoaortic balloon. We have found that this approach allows simpler cannulation, more rapid institution of cardiopulmonary bypass, and a more limited incision. In addition, use of the endoaortic balloon allows for ease of operation in a reoperative scenario. Although concerns regarding vascular complications exist for femoral cannulation, in our extensive experience we have noted very low rates of aortic injury with the use of the endoaortic balloon and femoral cannulation. ${ }^{3}$ Similar to both our study and that of the Columbia group, Grossi and colleagues ${ }^{6}$ analyzed the "Premier Database" containing patient billings and costs from more than 600 hospitals and found a shorter hospital stay (reduction of 2 days) with a $17 \%$ cost reduction 
associated with thoracotomy mitral valve surgery. Although specific details are difficult to ascertain and compare from this large, multicenter study, the trends corroborate findings from both this study and that of the Columbia group. ${ }^{5}$

In this study, we have demonstrated equivalent outcomes with both standard and minimally invasive approaches. This corroborates our recent propensity-matched, cohort-based study, which demonstrated equivalent outcomes with minimally invasive and sternotomy approaches. ${ }^{7}$ There have been numerous studies from excellent, high-volume, minimally invasive mitral valve centers that similarly have noted shorter hospital stays with excellent long-term outcomes. $^{8-10}$

On the basis of this study and preceding reports, we advocate a minimally invasive approach to the mitral valve for appropriately selected patients. In the properly selected scenarios, shorter hospital and ICU stays and excellent outcomes can be provided to patients without added cost, as we have demonstrated. It is important to note that there can be a significant learning curve associated with minimally invasive and robotic mitral valve surgery, and as such surgeons must be honest with themselves and their patients with respect to their comfort and experience with minimally invasive mitral valve surgery. Cost, however, should not be a limiting factor in offering minimally invasive mitral valve surgery to patients.

\section{CONCLUSIONS}

Minimally invasive mitral valve surgery can be performed with overall equivalent cost and shorter hospital and ICU stays than traditional sternotomy. There is greater operative cost associated with minimally invasive mitral valve surgery, but this is offset by shorter ICU and hospital stays.

\section{Conflict of Interest Statement}

W.C.H. is a consultant for Edwards Lifesciences. All other authors have nothing additional to disclose with regard to commercial support.

\section{References}

1. Galloway AC, Schwartz CF, Ribakove GH, Crooke GA, Gogoladze G, Ursomanno P, et al. A decade of minimally invasive mitral repair: long-term outcomes. Ann Thorac Surg. 2009;88:1180-4.

2. Gammie JS, Zhao Y, Peterson ED, O'Brien SM, Rankin JS, Griffith BP. J. Maxwell Chamberlain Memorial Paper for adult cardiac surgery. Less-invasive mitral valve operations: trends and outcomes from the Society of Thoracic Surgeons Adult Cardiac Surgery Database. Ann Thorac Surg. 2010;90:1401-8. 1410.e1; discussion 1408-10.

3. Atluri P, Goldstone AB, Fox J, Szeto WY, Hargrove WC. Port access cardiac operations can be safely performed with either endoaortic balloon or Chitwood clamp. Ann Thorac Surg. 2014;98:1579-83; discussion 1583-4.

4. Atluri P, Woo YJ, Goldstone AB, Fox J, Acker MA, Szeto WY, et al. Minimally invasive mitral valve surgery can be performed with optimal outcomes in the presence of left ventricular dysfunction. Ann Thorac Surg. 2013;96:1596-601; discussion 1601-2.

5. Iribarne A, Easterwood R, Russo MJ, Wang YC, Yang J, Hong KN, et al. A minimally invasive approach is more cost-effective than a traditional sternotomy approach for mitral valve surgery. J Thorac Cardiovasc Surg. 2011;142:1507-14.

6. Grossi EA, Goldman S, Wolfe JA, Mehall J, Smith JM, Ailawadi G, et al. Minithoracotomy for mitral valve repair improves inpatient and postdischarge economic savings. J Thorac Cardiovasc Surg. 2014;148:2818-22. e1-3.

7. Goldstone AB, Atluri P, Szeto WY, Trubelja A, Howard JL, MacArthur JW Jr, et al. Minimally invasive approach provides at least equivalent results for surgical correction of mitral regurgitation: a propensity-matched comparison. J Thorac Cardiovasc Surg. 2013;145:748-56.

8. Modi P, Rodriguez E, Hargrove WC III, Hassan A, Szeto WY, Chitwood WR Jr. Minimally invasive video-assisted mitral valve surgery: a 12-year, 2-center experience in 1178 patients. J Thorac Cardiovasc Surg. 2009;137:1481-7.

9. Borger MA, Kaeding AF, Seeburger J, Melnitchouk S, Hoebartner M, Winkfein M, et al. Minimally invasive mitral valve repair in Barlow's disease: early and long-term results. J Thorac Cardiovasc Surg. 2014;148:1379-85.

10. Suri RM, Antiel RM, Burkhart HM, Huebner M, Li Z, Eton DT, et al. Quality of life after early mitral valve repair using conventional and robotic approaches. Ann Thorac Surg. 2012;93:761-9.

Key Words: minimally invasive, mitral valve, outcomes, costs, health care economics 